\title{
Thymic cancer superimposed opacity of the mediastinal anatomical structures
}

\author{
Masahiro Uchiyama, Yuika Sasatani, Shinichiro Okauchi, Kesato Iguchi, \\ Norio Takayashiki, Hiroaki Satoh \\ Mito Medical Center, University of Tsukuba-Mito Kyodo General Hospital, Tsukuba, Japan
}

Thymic cancer is a rare malignant neoplasm. Among epithelial thymic tumors, those in which thymocyte atypia is apparent, are classified as thymic cancers [1]. Thymic cancer is highly malignant and has a high frequency of distant organ metastasis. In addition, it tends to infiltrate the surrounding organs and often forms a large tumor at the time of detection. Therefore, the majority of patients can detect a large tumor by chest radiograph. We show herein a patient with thymic cancer, which was superimposed on the opacities of mediastinal structures.

A 72-year-old woman visited our hospital complaining about discomfort in the chest. She was diagnosed as having hypertension several years ago and treated with antihypertensive drugs. Echocardiography revealed pericardial fluid. Chest radiograph at this visit showed cardiomegaly, but this finding was not documented in chest radiograph taken half a year ago (Figure 1). No tumor shadow was detected in the lung field, pulmonary hilum or mediastinum. Chest computed tomography (CT) scan showed an irregularly shaped mass measuring $3 \mathrm{~cm}$ in diameter, which was superimposed on the opacity of mediastinal structures, in the left anterior mediastinum, and CT scan also revealed multiple liver masses, which were evaluated as liver metastases (Figure 2). Since obtaining sufficient tissue samples by transbronchial biopsy was assessed as difficult, video-assisted thoracic surgery (VATS) was performed. On macroscopic findings, the tumor was identified as mediastinal tumor. Specimens from the mediastinal mass obtained by VATS proved to be a squamous cell carcinoma.
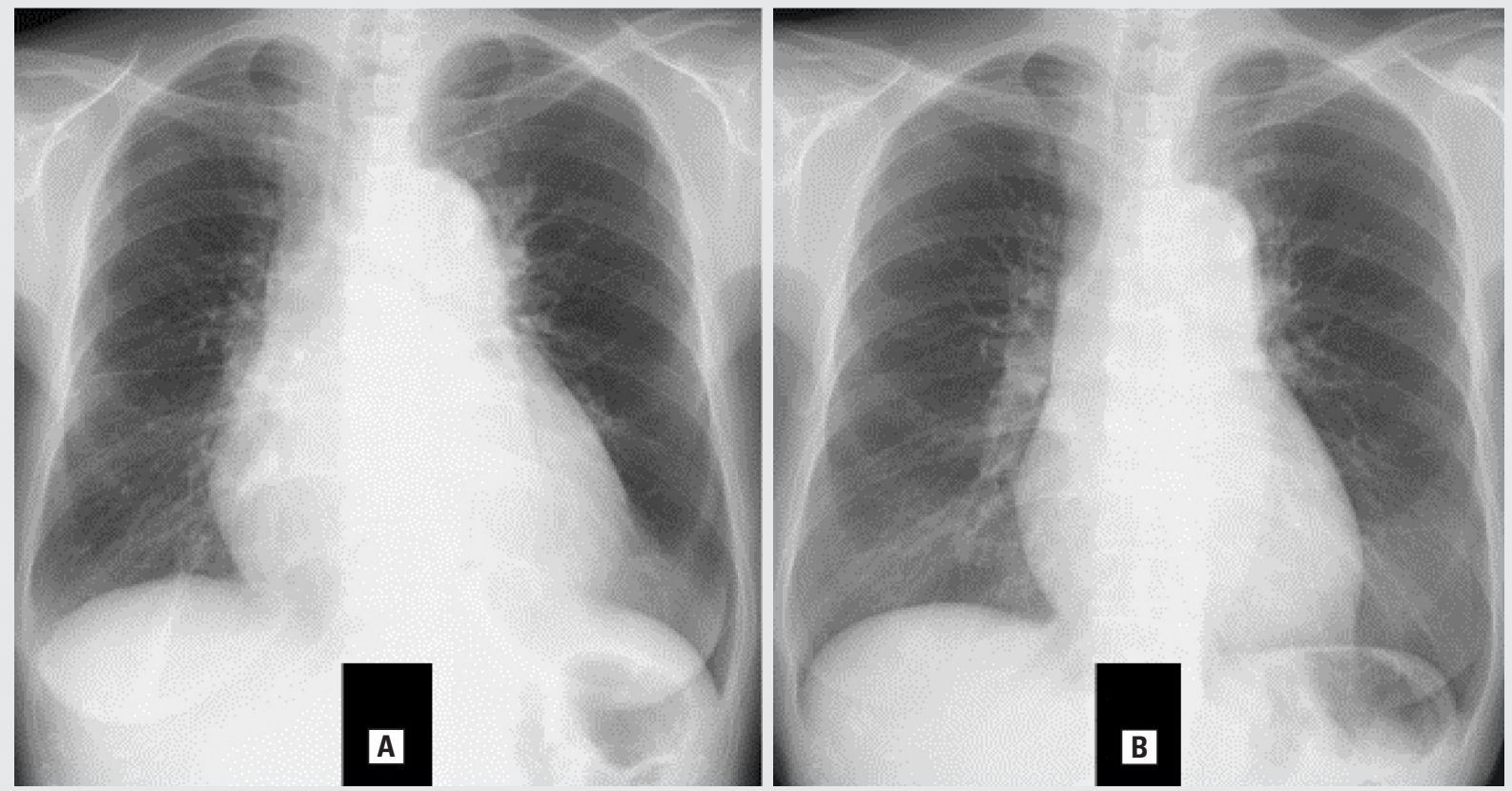

Figure 1. Chest radiograph at this visit showed cardiomegaly and abnormal separation of distance between the left hemidiaphragm and gastric air bubble (A), but this finding was not shown in chest radiograph taken half a year ago (B)

Address for correspondence: Hiroaki Satoh, Mito Medical Center, University of Tsukuba-Mito Kyodo General Hospital, Tsukuba, Japan; e-mail: hirosato@md.tsukuba.ac.jp DOI: 10.5603/ARM.a2020.0184

Received: 09.08.2020

Copyright (C) 2021 PTChP

ISSN 2451-4934

Conflict of interest: None declared 

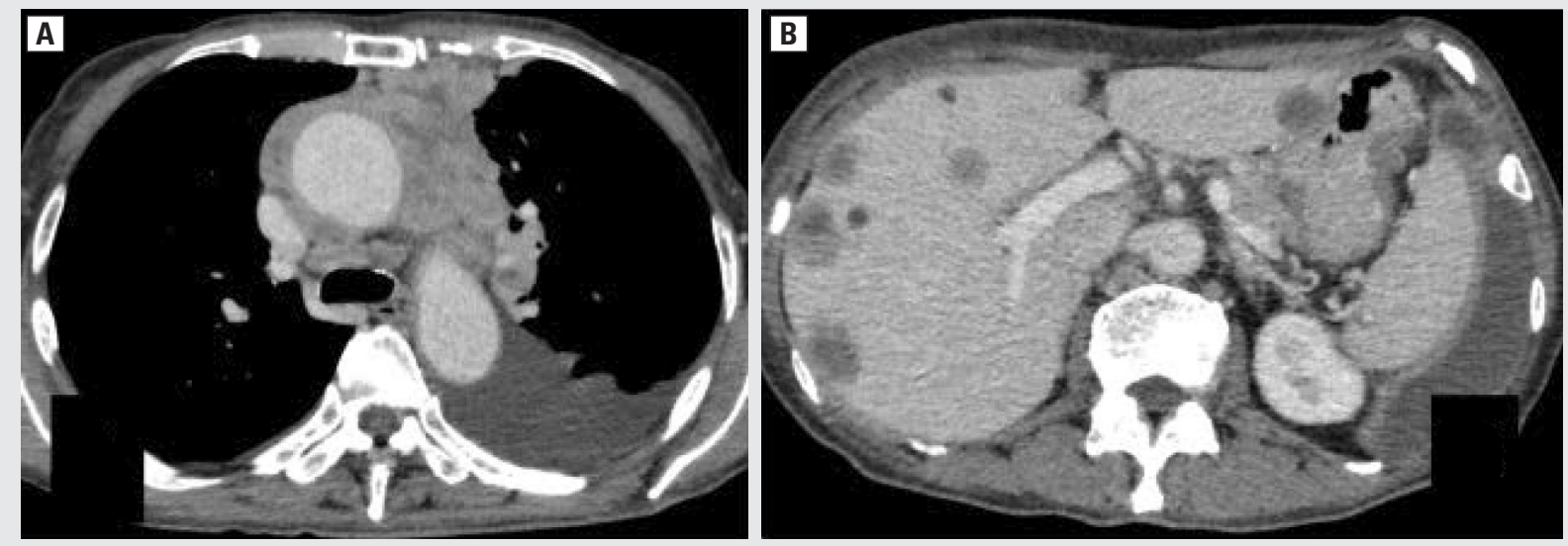

Figure 2. Chest CT scan showed an irregular-shaped mass measuring $3 \mathrm{~cm}$ in diameter, which was superimposed by the opacity of mediastinal structures, in the left anterior mediastinum (A). Abdominal CT scan revealed multiple liver masses, which were evaluated as metastatic liver tumors (B)

She was diagnosed as having thymic cancer with liver metastases, and received platinum-containing chemotherapy.

Although thymic cancer is a rare epithelial neoplasm, it tends to be aggressive and metastasizes widely. Many patients with thymic cancer already have distant organ metastases at the time of diagnosis. In addition, thymic cancers tend to invade surrounding organs, and often form large tumors. Therefore, the majority of thymic cancers can be easily detected as mediastinal masses on chest radiographs. The chest radiograph plays a pivotal role in the first-line imaging for initial detection of tumors originating from the thorax, although imaging for staging of most cancers is mainly based on CT scan. Regarding mediastinal tumors that could not be identified by chest radiograph, there were case reports on cardiac tumors [2, 3], esophageal submucosal tumor [4], and thymus-derived tumors [5, 6]. Thymolipoma is known as one of the superimposed thymus-derived tumors [5, 6]. Tindall et al. reported a patient with small thymolipoma which overlapped the mediastinum structures [5]. Mohan showed a patient with thymolipoma simulating cardiomegaly, an enlarged cardiac silhouette on chest radiograph. Chest CT revealed a giant anterior mediastinal non-contrast enhancing mass partially wrapping around the heart [6]. As far as we could search, however, there was no patient with thymic cancer, which was superimposed on mediastinal structures in chest radiograph. Although very rare, there are some mediastinal tumor patients with undetected primary lesion on chest radiograph. In such cases, chest CT is useful in confirming the exact extent of the mediastinal tumor, which is superimposed on opacities of mediastinal structures.

\section{References:}

1. Shimosato Y, Kameya T, Nagai K, et al. Squamous cell carcinoma of the thymus. An analysis of eight cases. Am J Surg Pathol. 1977; 1(2): 109-121, doi: 10.1097/00000478-197706000-00002, indexed in Pubmed: 602973.

2. Hsueh SC, Chung MT, Fang R, et al. Primary cardiac lymphoma. J Chin Med Assoc. 2006; 69(4): 169-174, doi: 10.1016/S17264901(09)70200-X, indexed in Pubmed: 16689199.

3. Vakili H, Khaheshi I, Memaryan M, et al. Angiosarcoma of the Right Atrium with Extension to SVC and IVC Presenting with Complete Heart Block and Significant Pericardial Effusion. Case Rep Cardiol. 2016; 2016: 3173069, doi: 10.1155/2016/3173069, indexed in Pubmed: 27110407.

4. Shang QX, Yang YS, Wang WP, et al. Missed diagnosis of esophageal leiomyoma leading to esophagectomy: a case report and review of literatures. J Thorac Dis. 2018; 10(1): E65-E69, doi: 10.21037/jtd.2017.12.05, indexed in Pubmed: 29600107.

5. Tindall H, Tandon AP, Ionescu MI. An unusual case of chest pain and cardiomegaly. Br J Clin Pract. 1990; 44(12): 766-767, indexed in Pubmed: 2151679.

6. Mohan Rao PS, Moorthy N, Shankarappa RK, et al. Giant mediastinal thymolipoma simulating cardiomegaly. J Cardiol. 2009; 54(2): 326-329, doi: 10.1016/j.jicc.2008.12.008, indexed in Pubmed: 19782275. 